INVESTIGACIÓN

https://doi.org/10.15198/seeci.2020.53.135-151

Recibido: 30/05/2020 --- Aceptado: 07/09/2020 --- Publicado: 15/11/2020

\title{
LIDERAZGO TRANSFORMACIONAL Y DESARROLLO SOSTENIBLE AMBIENTAL VERDE EN DOCENTES DE LA UNIVERSIDAD NACIONAL HERMILIO VALDIZÁN
}

\section{TRANSFORMATIONAL LEADERSHIP AND SUSTAINABLE GREEN ENVIRONMENTAL DEVELOPMENT IN TEACHERS AT HERMILIO VALDIZAN NATIONAL UNIVERSITY}

Atanacia Santacruz Espinoza1: Universidad Nacional Intercultural de la Amazonia. Perú.

atanacia.santacruz@gmail.com

Humberto Montenegro Muguerza: Universidad Nacional Hermilio Valdizán. Perú.

catedraHMM1@hotmail.com

Armando Pizarro Alejandro: Universidad Nacional Hermilio Valdizán. Perú. pizarro1905@hotmail.com

Hamilton Estacio Flores: Universidad Nacional Hermilio Valdizán. Perú. hamilton.e.s@hotmail.com

\section{RESUMEN}

El concepto desarrollo sostenible verde articula las diversas disciplinas científicas del saber actuar, pensar, vivir, sentir. Por ende, el líder transformador debe propiciar el cambio de una economía negra a una economía ambiental verde. Por ende, garantizar los derechos naturales a través del empoderamiento sostenible en el tiempo. La investigación fue aplicada, nivel explicativo, y diseño descriptivocorrelacional, con un muestreo probabilístico y estratificado. Por su alcance es descriptiva y por su finalidad cuantitativa, cualitativa y mixto. Metodológicamente, la investigación pone en diálogo fuentes bibliográficas diversas. Se ha realizado el análisis de los resultados utilizando los estadígrafos inferencial y descriptiva. El método utilizado es el método científico y como métodos específicos: hermenéutico, dogmático y heurístico. Se utilizó la técnica de encuesta y el instrumento cuestionario de encuesta. Como principal conclusión se tiene que existe una correlación directa fuerte ( $r$ ho $=0,788$ ) y altamente significativo con ( $p$-valor: 0,000 ) entre el liderazgo transformacional y el desarrollo sostenible verde Los estudiantes, docentes y administrativos de la Universidad nacional Hermilio Valdizán tuvieron un nivel medio

\footnotetext{
${ }^{1}$ Atanacia Santacruz Espinoza. Docente de la Universidad nacional Intercultural de la Amazonia
} 
Santacruz Espinoza, A., Montenegro Muguerza, H., Pizarro Alejandro, A. y Estacio Flores, H. Liderazgo transformacional y desarrollo sostenible ambiental verde en docentes de la

Universidad Nacional Herminio Valdizán

o regular, se ha descubierto en ellos que dan orientación a la sociedad para el reciclado adecuado, propicia conversatorios para no pagar elevados precios por el consumo de electricidad, comprar solo lo que se necesita, dejar de comprar alimentos chatarra en pro de la salud, utilizar el transporte de la universidad o público en vez de privado; ello, para la sostenibilidad generacional con práctica de una economía circular a través del uso de tecnología responsable.

PALABRAS CLAVE: Liderazgo transformacional - Desarrollo sostenible verde Gestión de recursos - Actitud económica - Innovación ambiental verde

\section{ABSTRACT}

The green sustainable development concept articulates the various scientific disciplines of knowing how to act, think, live, feel. Therefore, the transformative leader must promote the change from a black economy to a green environmental economy. Therefore, guarantee natural rights through sustainable empowerment over time. The research was applied, explanatory level, and descriptive-correlational design, with a probabilistic and stratified sampling. Due to its scope it is descriptive and due to its quantitative, qualitative and mixed purpose. Methodologically, the research puts various bibliographic sources into dialogue. The analysis of the results has been carried out using the inferential and descriptive statistics. The method used is the scientific method and as specific methods: hermeneutic, dogmatic and heuristic. The survey technique and the survey questionnaire instrument were used. The main conclusion is that there is a strong (rho $=0.788)$ and highly significant direct correlation with ( $p$-value: 0.000 ) between transformational leadership and green sustainable development The students, teachers and administrators of the Hermilio Valdizán National University had a medium or regular level, it has been discovered in them that they give society guidance for proper recycling, conducive discussions to avoid paying high prices for electricity consumption, buy only what is needed, stop buying junk food in favor of health, use university or public transportation instead of private; This, for the generational sustainability with the practice of a circular economy through the use of responsible technology.

KEY WORDS: Transformational leadership - Green sustainable development Resource management - Economic attitude - Green environmental innovation

\section{LIDERANÇA TRANSFORMACIONAL E DESENVOLVIMENTO SUSTENTÁVEL AMBIENTAL VERDE EM PROFESSORES DA UNIVERSIDADE NACIONAL HERMILIO VALDIZÁN}

\section{RESUMO}

O conceito desenvolvimento sustentável verde articula as diversas disciplinas científicas do saber atuar, pensar, viver, sentir. Portanto, o líder transformador deve propiciar as mudanças de uma economia destrutiva à uma economia ambiental verde. Portanto, garantir os direitos naturais através do empoderamento sustentável 
Santacruz Espinoza, A., Montenegro Muguerza, H., Pizarro Alejandro, A. y Estacio Flores, H. Liderazgo transformacional y desarrollo sostenible ambiental verde en docentes de la

Universidad Nacional Herminio Valdizán

no tempo. A pesquisa foi aplicada, a nível explicativo, e de forma descritivacorrelacional, com uma amostragem probabilística e estratificada. Pelo seu alcance é descritiva e pela sua finalidade quantitativa, qualitativa e mista. Metodologicamente, a pesquisa coloca em diálogo fontes bibliográficas diversas. Foi feita uma análise dos resultados usando os gráficos estadísticos inferencial e descritivo. O método utilizado é o método científico e como métodos específicos: hermenéutico, dogmático e heurístico. Utilizou-se a técnica de questionário e seus instrumentos. Como principal conclusão temos que existe uma correlação direta forte (rho $=0,788$ ) e altamente significativa ( $p$-valor: 0,000) entre a liderança transformacional e 0 desenvolvimento sustentável verde dos estudantes, professores e trabalhadores administrativos da Universidade nacional Hermilio Valdizán que tiveram um nível médio ou regular, foi encontrado neles que dão orientação a sociedade para a reciclagem adequada, encorajam conversas para não pagar elevados preços pelo consumo elétrico, comprar somente o que é preciso, deixar de comprar alimentos não saudáveis para favorecer a saúde, usar o transporte da universidade ou público em vez de privado; isto, para a sustentabilidade geracional com prática de uma economia circular através do uso de tecnología responsável.

PALAVRAS CHAVE: Liderança transformacional - Desenvolvimento sustentável verde - Gestão de recursos - Atitude economica - Inovação ambiental verde

\section{Cómo citar el artículo:}

Santacruz Espinoza, A., Montenegro Muguerza, H., Pizarro Alejandro, A. y Estacio Flores, H. (2020). Liderazgo transformacional y desarrollo sostenible ambiental verde en docentes de la Universidad Nacional Herminio Valdizán. [Transformational leadership and sustainable green environmental development in teachers at Hermilio Valdizan National University]. Revista de Comunicación de la SEECI, 53, 135-151. doi: https://doi.org/10.15198/seeci.2020.53.135-151

Recuperado de http://www.seeci.net/revista/index.php/seeci/article/view/649

\section{INTRODUCCIÓN}

La educación juega un papel muy importante en la sociedad peruana y global debido al impacto ambiental en la cultura de consumo colectiva de hoy. Lo importante es incrementar educación en el campo de sostenibilidad, los estudiantes deben estar informados de sostenibilidad, esto se aplica a través de acciones proambientales, puesto que el hábito se desarrolla desde temprana edad, desde jugando, hablando, comiendo, durmiendo, aplaudiendo, riendo; se debe gestionar, implementar en el estilo de vida diario de los estudiantes, profesionales, y la sociedad para que practiquen las acciones en el fortalecimiento del pensamiento crítico y solución de problemas en materia de desarrollo sostenible verde. Así como Habit (2017), citado en (Gardner, 2017) sustenta que el trabajo sostenible se logra a través de la educación de la sostenibilidad y se aplica a través de acciones prosostenibilidad. Dado que el hábito puede ser parte de cualquier actividad, desde comiendo y durmiendo para pensar y reaccionar a través del refuerzo y la repetición, los comportamientos de sostenibilidad deben implementarse en el estilo de vida 
Santacruz Espinoza, A., Montenegro Muguerza, H., Pizarro Alejandro, A. y Estacio Flores, H. Liderazgo transformacional y desarrollo sostenible ambiental verde en docentes de la Universidad Nacional Herminio Valdizán

diario de los estudiantes para que puedan continuar estas acciones a medida que se convierten en miembros críticos de la sociedad.

Los problemas ambientales constituyen una dimensión global, o sea compromete a todo el planeta, afecta a todos los ecosistemas como consecuencia del desarrollo de la tecnología y la industrialización de los países que buscan su crecimiento económico, es por ello, que algunas organizaciones económicas internacionales de primera línea, como el Banco Mundial y la Organización para la Cooperación y el Desarrollo Económico (OCDE), ratifican estas preocupantes predicciones. Por ejemplo, la OCDE ha presentado una relación muy desalentadora de los retos a los que se enfrenta la humanidad: lucha contra el cambio climático, detención de la pérdida de biodiversidad, abastecimiento de agua dulce, garantía de una sanidad pública adecuada y reducción de los impactos del deterioro del medio ambiente sobre la salud de las personas (OCDE, 2011).

Del mismo modo, los estados de América y el mundo han tocado la problemática del medio ambiente no en su verdadera dimensión para afrontar en sus situaciones reales y contundentes; nos da la impresión que los fenómenos naturales se han adelantado a las investigaciones que realiza el hombre y que en el mayor de los casos el hombre se siente impotente en su propósito de evitar y/ o atenuar el deterioro de los ecosistemas y de la biodiversidad. La afirmación de poder manejar la naturaleza y otorgarle poca importancia al equilibrio de los recursos naturales está cambiando rápidamente ante los insistentes avisos de la madre Tierra.

Este proceso de cambio se sustenta en un dar una mirada rápida del detrimento del ambiente, producto del irracional manejo de los recursos naturales renovables, por la acelerada contaminación del aire, mares, ríos y suelos, en la constante información sobre el ambiente que llega e impacta a través de las tecnologías de la información y en los programas de educación ambiental insertados en la mayoría de los currícula a nivel mundial y en todos los niveles de educación.

La Agenda 21 hace hincapié que la educación debe:

- Crear conciencia sobre el medio ambiente y el desarrollo en todos los sectores de la sociedad a escala mundial y a la mayor brevedad posible.

- Procurar, facilitar el acceso a la educación sobre el Medio Ambiente y el desarrollo, vinculada con la educación social, desde la edad escolar hasta la edad adulta en todos los grupos de población.

- Las universidades deben estimular la participación de los estudiantes en estudios locales y regionales sobre salud ambiental, agua potable, saneamiento, alimentación, ecosistemas, fauna y flora, etc.

En el 2015, las Naciones Unidas lanzaron los Objetivos de Desarrollo Sostenible (ODS), lo que constituye una oportunidad histórica y sin precedentes para unir a los países y a las personas de todo el mundo. Los ODS permitirán determinar el curso de las medidas destinadas a erradicar la pobreza, promover la prosperidad y el bienestar para todos, proteger el medio ambiente y hacer frente al cambio climático a nivel mundial. 
Santacruz Espinoza, A., Montenegro Muguerza, H., Pizarro Alejandro, A. y Estacio Flores, H. Liderazgo transformacional y desarrollo sostenible ambiental verde en docentes de la Universidad Nacional Herminio Valdizán

Las Naciones Unidas en su informe de los Objetivos del Milenio (ODM, 2017) sostiene que el Objetivo $\mathrm{N}^{\circ} 7$ referido a "garantizar la sostenibilidad del medio ambiente" cuya meta consiste en incorporar los principios del Desarrollo Sostenible en las políticas y programas nacionales e invertir pérdida de recursos del medio ambiente.

Se necesita con urgencia comenzar con el ser humano como ente individual y colectivo: determinar los niveles de liderazgo transformacional y desarrollo sostenible ambiental verde. Se debe tomar conciencia que la gestión es importante para entender lo que hacemos, pero la medición de nuestros impactos es los que determinarán la sostenibilidad del planeta. Nuestra forma de vida, nuestros estilos de consumos, nuestros hábitos; son estos los que definen la sostenibilidad.

En verdad, es hora la asunción de responsabilidades sobre un proceso; es decir, sobre un conjunto de actividades en favor de la sostenibilidad ambiental y convertirnos en agentes activos de la defensa del planeta y no pobladores del mundo que ven con indiferencia como se desvanece ante sus ojos el legado natural de las nuevas generaciones.

En el departamento de Huánuco, se ha podido observar de manera directa que carecen de actitudes en favor de la conciencia ambiental, ya que es común encontrar en el patio de las instituciones educativas, en las aulas, en los servicios higiénicos, desechos o basuras en los pisos; en las calles, etc.; es más, se incinera la basura a vista y paciencia de la sociedad. Asimismo, se ha podido observar el agotamiento del agua gota a gota. Además, se deduce que no hay conciencia de plantar un árbol ni mucho menos sembrar una semilla para el bien común, puesto que algunas instituciones educativas no las tiene. Sobre lo expresado, (Brack, 2012), dice: La contaminación del agua, causada fundamentalmente por las aguas servidas que las ciudades y pueblos tiran. El segundo problema es la disposición inadecuada de residuos sólidos... La ciudad está generando un nuevo tipo de ciudadano con un enfoque totalmente distinto y que no comprende la naturaleza porque no la ha vivido.

En resumen, es imprescindible comprender que los problemas ambientales son muy complejos y requieren un enfoque interdisciplinario que permita atender las necesidades de una población aumentada en las próximas décadas frente al desafío de una capacidad operativa para gestionar y restaurar los recursos naturales de los que depende toda la vida (Bartolotta, 2015). La universidad cumple un rol muy importante a través de la responsabilidad social universitaria, en las dimensiones académicas, de investigación, de participación en el desarrollo social y servicios de extensión ambiental e institucional. Como se visualiza, nadie puede manejar sus impactos por sí solo; siempre los impactos son sociales. Por lo tanto, de forma individual no se logrará el cambio, si lo hacemos de forma sistémica sí. Montenegro (2019). 
Santacruz Espinoza, A., Montenegro Muguerza, H., Pizarro Alejandro, A. y Estacio Flores, H. Liderazgo transformacional y desarrollo sostenible ambiental verde en docentes de la Universidad Nacional Herminio Valdizán

\subsection{Liderazgo}

Según la Real Academia de la Lengua Castellana-RAE (2018), liderazgo es el ejercicio de las actividades del líder. Por su parte Goleman (2013) dice que, el liderazgo es el arte de convencer a la gente de que colabore para alcanzar un objetivo común. Además, centrándonos en la gestión de nuestra trayectoria personal, puede que no haya nada más fundamental que reconocer una profunda conexión emocional con nuestro cometido y saber qué cambios podrían provocarnos una mayor satisfacción laboral. Mientras que para (Drucker, 1993) el liderazgo es el desempeño, cada integrante cumple con sus responsabilidades.

El liderazgo es el desarrollo de un sistema completo de expectativas, capacidades y habilidades que permiten identificar, descubrir, utilizar, reutilizar, potenciar y estimular al máximo la fortaleza y la energía de todos los recursos humanos de la organización, que incrementan la productividad, eficiencia, innovación y creatividad, permitiendo el éxito organizacional y la satisfacción de las necesidades de todos los inquilinos del planeta Tierra.

Lussier y Achua (2016) sostienen que: "El liderazgo es el proceso de influencia entre líderes y seguidores para lograr los objetivos de la organización por medio del cambio". Por su parte, Pozueta (2015) afirma que el Liderazgo se basa en el desarrollo de las emociones clave que todo líder debe tener para influir, desarrollar, animar, identificar y lanzar a su equipo hacia la consecución de sus metas y objetivos.

En síntesis, el liderazgo es la capacidad de tomar decisiones, gestionar, promover, incentivar, motivar, delegar, coordinar, monitorear y evaluar al equipo de trabajo en favor de los objetivos a alcanzar en una determinada organización, sobre todo con el cambio de actitudes, capacidad de escucha y mucha comunicación. Ya que, el liderazgo es asunto de todos para la solución de conflictos en la diversidad de problemas.

\subsection{Liderazgo transformacional}

Para Lussier y Achua (2016), los líderes transformacionales son conocidos por conmover y cambiar las cosas en "gran forma", al comunicar a los seguidores una visión especial del futuro, utilizando los ideales y motivos más altos; influir para promover la idea de una visión y posibilidades nuevas conjuntamente con los seguidores. En cambio, Jordán y Garay (2014), señala que, "el liderazgo transformacional se sustenta en un compromiso ético colectivo que se produce cuando las personas se animan unas a otras a pasar a estadios superiores de motivación y convivencia".

En conclusión, el liderazgo transformacional es el proceso en el que un individuo promueve y crea conexiones entre ellos generando una red organizacional para elevar la motivación y la moral en forma conjunta en el logro de acuerdos sostenibles en temas ambientales, culturales, sociales, políticas y lingüísticas. 
Santacruz Espinoza, A., Montenegro Muguerza, H., Pizarro Alejandro, A. y Estacio Flores, H. Liderazgo transformacional y desarrollo sostenible ambiental verde en docentes de la Universidad Nacional Herminio Valdizán

\subsection{Factores de liderazgo transformacional}

Los factores del liderazgo transformacional, aluden a un conjunto de elementos necesarios para lograr la configuración e implementación del mismo en el ambiente institucional. Bass y Avolio, (2006a) logran resultados en una o más de las siguientes maneras: son carismáticos a los ojos de sus seguidores y son una fuente de inspiración para ellos; pueden tratar individualmente para satisfacer las necesidades de cada uno de sus subordinados; y pueden estimular intelectualmente a sus subordinados. Estos factores representan los cuatro componentes básicos del liderazgo transformacional: a) Influencia idealizada (Liderazgo Carismático): Los líderes tienen una visión y sentido de misión; que se ganan el respeto, confianza y seguridad; y que adquieren una identificación individual de sus seguidores. Los líderes de influencia idealizada son capaces de obtener el esfuerzo extra requerido de los seguidores para lograr niveles óptimos de desarrollo y desempeño. b) Consideración individualizada: Estos líderes se concentran en diagnosticar las necesidades y capacidades de los seguidores. Precisan las necesidades de los seguidores y atienden a ellas individualmente. También delegan, entrenan, aconsejan y proveen retroalimentación para el uso en el desarrollo personal de los seguidores. Elevan el nivel de necesidad y seguridad de los discípulos para adquirir mayores niveles de responsabilidad. Las responsabilidades de los seguidores adquieren mayor responsabilidad para su desarrollo personal, que pueden incluir tales actividades como los desafíos del trabajo mismo. c) Estimulación intelectual: Los líderes activamente fomentan una nueva mirada en cuanto a métodos y problemas. Fomentan la creatividad, y enfatizan un repensamiento y reexaminación de suposiciones subyacentes a los problemas. Utilizan la intuición, así como una lógica más formal para solucionar los problemas. Los líderes que estimulan intelectualmente desarrollan seguidores que atacan los problemas usando sus propias perspectivas únicas e innovativas. Los seguidores se transforman en solucionadores de problemas más efectivos con y sin la facilitación del líder. Llegan a ser más innovativos con respecto a su análisis de problemas y de las estrategias que usan para resolverlos. d) Liderazgo inspiracional: Los líderes dan ánimo, aumentan el optimismo y entusiasmo, y comunican sus visiones de futuros realizables con fluidez y seguridad (Bass, 1985). Provee visión, la cual estimula la energía para lograr altos niveles de desempeño, pero la comunicación es muy importante en la adherencia de las prácticas para el buen vivir.

\subsection{Desarrollo sostenible}

Según el Informe Brundtland (1987) el desarrollo sostenible es aquel que satisface las necesidades del presente sin limitar el potencial para satisfacer las necesidades de las generaciones futuras.

Sin duda alguna, Bernard (1999) sostiene que el desarrollo sostenible es un sistema o proceso que puede continuar indefinidamente sin agotar nada de los recursos materiales o energéticos que necesita para funcionar. El vocablo se utilizó por primera vez en términos de producción sostenible en desempeños humanos 
Santacruz Espinoza, A., Montenegro Muguerza, H., Pizarro Alejandro, A. y Estacio Flores, H. Liderazgo transformacional y desarrollo sostenible ambiental verde en docentes de la Universidad Nacional Herminio Valdizán

como la silvicultura y la pesca. Estas ideas primigenias han dado origen a un vasto campo de interdisciplinaridad en la sostenibilidad.

Concretizando, el desarrollo sostenible gestiona la protección, conservación y mejoramiento de la calidad de vida en la sociedad en interacción con la Naturaleza. Implica conciencia, sensibilidad, responsabilidad social, económica, política, cultural, intercultural, y responsabilidad universitaria; así como, patrones de consumo y estilos de vida, aplicado al desarrollo sin poner en peligro la capacidad de futuras generaciones para satisfacer sus propias necesidades y disfrutar de la felicidad ecológica.

\subsection{La economía verde/crecimiento verde}

La economía verde se precisa como aquella que se transcribe en "la mejora del bienestar humano y la equidad social, reduciendo significativamente los riesgos ambientales y las escaseces ecológicas" (Becker, 2017).

La Organización para la Cooperación y el Desarrollo Económico - OCDE, (2011) crecimiento verde significa fomentar el crecimiento y desarrollo económicos y al mismo tiempo asegurar que los bienes naturales continúen proporcionando los recursos y los servicios ambientales de los cuales depende nuestro bienestar. Para lograrlo debe catalizar inversión e innovación que apuntalen el crecimiento sostenido y abran paso a nuevas oportunidades económicas.

Requerimos el crecimiento verde, ya que los riesgos para el desarrollo se van incrementando el deterioro del bien común ambiental. Si el problema no es controlado, significa una mayor escasez de agua, tierras agrícolas improductivas, una desmedida contaminación, cambio climático y una pérdida irreversible de la megadiversidad.

Estas tensiones pueden debilitar las perspectivas de crecimiento verde. Cada vez es más costoso sustituir el capital físico por capital natural. Por ejemplo, si el agua escasea o se contamina, se necesitará más procesos para purificarla para el consumo humano. Se tiene que encontrar nuevas maneras de producir y consumir, e inclusive redefinir lo que queremos decir con el término progreso y cómo lo medimos.

Es necesario aclarar, como señalan (Chankrajang y Muttarak, 2017) parte de ese progreso, se debió a las actividades industriales (orientadas a la exportación), los cambios en el estilo de vida, el consumo y los patrones de movilidad también jugaron un papel significativo. Cómo las sociedades logran responder a las presiones ambientales depende en gran medida de comportamiento de los seres humanos que actúan individual o colectivamente. En consecuencia, promover un estilo de vida sostenible y el consumo es una estrategia clave para reducir el impacto humano sobre el clima en los países en desarrollo, especialmente dado el hecho de que son estos países los que más sufren el impacto del medioambiente global. 
Santacruz Espinoza, A., Montenegro Muguerza, H., Pizarro Alejandro, A. y Estacio Flores, H. Liderazgo transformacional y desarrollo sostenible ambiental verde en docentes de la Universidad Nacional Herminio Valdizán

Como una alternativa de solución, "estudios recientes han demostrado que promover la educación universal puede ser un medio efectivo no solo para aliviar la pobreza y para fomentar el crecimiento económico en los países en desarrollo, pero también para reducir la vulnerabilidad al cambio climático" (Lutz, Muttarak y Striessnig, 2014). Del mismo modo, siguen afirmando (Lutz y Muttarak, 2014) que puede ser eficiente y efectivo dar parte de este fondo a educadores en lugar de ingenieros. La inversión pública en educación universal en los países pobres en el futuro cercano debe verse como una de las principales prioridades para mejorar la capacidad de adaptación de las sociedades frente al cambio climático.

\subsection{Estrategias de crecimiento verde}

Para la OCDE (2011) las estrategias de crecimiento verde, la meta es establecer incentivos o instituciones que aumenten bienestar al:

- Mejorar la gestión de recursos e implementar la productividad.

- Propiciar que la actividad económica tenga lugar donde sea más ventajoso para la sociedad a largo plazo.

- Conducir a nuevas maneras de cumplir a través de la innovación.

En tal sentido, el crecimiento de una economía verde depende de los escenarios institucionales y de políticas, el grado de desarrollo, la disponibilidad de recursos y los aspectos particulares de presión ambiental. Los países avanzados, emergentes y en desarrollo afrontan diversos retos y oportunidades al dar a su crecimiento un enfoque verde. Por otro lado, existen consideraciones que deben abordarse en todos los escenarios, OCDE (2011).

Dentro de esta orden de ideas (Meyer, 2015) apuesta por una educación, las personas en la educación superior están más educada en ahorro financiero, por eso participan en acciones más conscientes de la sostenibilidad. (Hwang, Park y Kim, 2016) Aunque las etiquetas ecológicas se introdujeron con la intención de fomentar un comportamiento de compra ecológico por parte de los consumidores, han tenido poco efecto en las decisiones de compra de los consumidores y, por lo tanto, existe una brecha significativa entre el conocimiento de la etiqueta ecológica y el comportamiento real de compra (...) los resultados indican que, aunque los consumidores en general son muy conscientes de la información públicamente valiosa que proporcionan las etiquetas ecológicas, la información de valor privado ejerce un poder mucho mayor sobre sus intenciones de compra. Por lo tanto, una política complementaria que convierta el valor público en valor privado podría promover la compra de productos con etiqueta ecológica. Quien realizó un análisis teórico y relacionó dos enfoques: experimental e instructivo, obteniendo el enfoque combinado. La idea de dicho enfoque se basa en la práctica directa con el ambiente, en la que se desarrollan significados mediante el contacto, por lo que se nutren con el conocimiento del sector. El carácter instructivo de dicho enfoque mantiene diferencias en torno a que el significado no se adquiere por el contacto con el ambiente, sino que el educando elabora un significado y lo transfiere a su ambiente. Además, el enfoque combinado surge de la aplicación experimental e instructivo, y el 
Santacruz Espinoza, A., Montenegro Muguerza, H., Pizarro Alejandro, A. y Estacio Flores, H. Liderazgo transformacional y desarrollo sostenible ambiental verde en docentes de la Universidad Nacional Herminio Valdizán

contacto recíproco de significados, del medio con el sujeto y el sujeto con el medio; y se utiliza el diálogo para dar énfasis a los significados.

Entonces, se ve cambios en cuanto a la actitud (Escobedo, 2015) sostiene que, con la ISO 14001: 2015, las empresas por su propia iniciativa cumplen una serie de obligaciones, quien la obtiene se diferencia de las demás y tiene muchas oportunidades. Pues, estas gestionan el impacto ambiental para obtener certificación.

\section{OBJETIVOS}

A través de las contribuciones de la bibliografía centrada en investigación de ambas variables. La pesquisa persigue un objetivo principal: Interpretar el nivel de relación entre el liderazgo transformacional y desarrollo sostenible verde en los docentes de la Universidad Nacional Hermilio Valdizan.

A partir del objetivo general se desarrolló tres objetivos secundarios:

- Interpretar el nivel de relación entre liderazgo transformacional y gestión de recursos ambiental verde en la Universidad Nacional Hermilio Valdizán.

- Interpretar el nivel de relación entre liderazgo transformacional y actividad económica ambiental verde en la Universidad Nacional Hermilio Valdizán.

- Interpretar el nivel de relación entre liderazgo transformacional y la innovación ambiental verde en la Universidad Nacional Hermilio Valdizán.

\section{METODOLOGÍA}

\subsection{Tipo de estudio}

La presente investigación tuvo como percepción el enfoque positivista de tipo descriptivo; a partir del análisis de cada una de las variables se establecieron relaciones con el propósito de identificar y explicar el nivel de relación existente entre ambas variables de estudio. Esta indagación constituyó un aporte teórico que permitió tener mayor claridad sobre el comportamiento de las variables (Kerlinger y Lee, 2002), el diseño utilizado en la investigación fue correlacional, para establecer la relación no causal entre las variables buscando determinar el nivel de relación entre ambas.

\subsection{Población}

Según, Oseda, Santacruz, Zevallos, Sangama, Cosme y Mendivel (2018), "la población involucra a total de sujetos a los que se intentará generalizar los resultados; es decir, constituida por todos los sujetos a los que tiene acceso el investigador".

La población estuvo conformada por 244 docentes de la Universidad Nacional Hermilio Valdizán. Según Kerlinger y Lee (2002) el diseño descriptivo correlacional es aquel diseño donde no se manipula ninguna variable, solo se miden y luego se compara para ver qué relación existen entre estas. 
Santacruz Espinoza, A., Montenegro Muguerza, H., Pizarro Alejandro, A. y Estacio Flores, H. Liderazgo transformacional y desarrollo sostenible ambiental verde en docentes de la Universidad Nacional Herminio Valdizán

\subsection{Procedimiento}

Se llevó a cabo las coordinaciones con los decanos de las distintas Carreras Profesionales para la recolección de datos. Luego con la autorización de la autoridad correspondiente se procedió a aplicarlos cuestionarios de encuesta en la muestra especificada. Esta recolección se realizó en los meses de setiembre, octubre y noviembre del 2019.

\section{RESULTADOS}

Para demostrar la hipótesis planteada, se presenta los datos obtenidos en las siguientes tablas e interpretaciones:

Tabla 1: Medición de los niveles de la V1: Liderazgo transformacional

\begin{tabular}{cccc}
\hline Niveles & & Frecuencia & Porcentaje \\
\hline & Muy alto & 18 & 7,38 \\
& Alto & 56 & 22,95 \\
Medio & 158 & 64,75 \\
Bajo & 12 & 4,92 \\
Muy bajo & 0 & 0,00 \\
Total & 244 & 100,00 \\
\hline
\end{tabular}

Fuente: Base de datos de los investigadores (2019)

Respecto a los niveles de la variable 1: Liderazgo transformacional en la Universidad Nacional Hermilio Valdizán, se tiene que la mayoría de ellos que representa el $64,75 \%$ estuvieron en el nivel medio, seguido del $22,95 \%$ en el nivel alto, luego $7,38 \%$ en el nivel muy alto, seguido el $4,92 \%$ el nivel bajo. Los cuales se aprecian en la tabla 2. Cabe mencionar que el nivel predominante diagnosticado es el nivel medio o regular, el cual es preocupante pues se trata de reflexionar sobre nuestra actuación, también se percibe que esta tendencia está en aumento hacia el nivel alto, el cual es favorable el empoderamiento de nuestras acciones.

Tabla 2: Medición de los niveles de la V2: Desarrollo sostenible verde

\begin{tabular}{cccc}
\hline Niveles & Frecuencia & Porcentaje \\
\hline & Muy alto & 10 & 4,10 \\
& Alto & 61 & 25,00 \\
Medio & 145 & 59,43 \\
Bajo & 28 & 11,48 \\
Muy bajo & 0 & 0,00 \\
Total & 244 & 100,00 \\
\hline
\end{tabular}

Fuente: Base de datos de los investigadores (2019) 
Santacruz Espinoza, A., Montenegro Muguerza, H., Pizarro Alejandro, A. y Estacio Flores, H. Liderazgo transformacional y desarrollo sostenible ambiental verde en docentes de la Universidad Nacional Herminio Valdizán

Respecto a los niveles de la variable 2: Desarrollo sostenible verde en la Universidad Nacional Hermilio Valdizán, se tiene que la mayoría de ellos que representa el 59,43\% estuvieron en el nivel medio, seguido del $25,00 \%$ en el nivel alto, luego $11,48 \%$ en el nivel muy alto, seguido el $4,92 \%$ el nivel bajo. Los cuales se aprecian en la tabla 3. Cabe mencionar que el nivel predominante diagnosticado es el nivel medio o regular, el cual es preocupante pues se trata de recapacitar sobre nuestra actuación, también se percibe que esta tendencia está en aumento hacia el nivel alto, el cual es favorable.

Tabla 3. Correlación de variables: Liderazgo transformacional y el desarrollo sostenible verde

\begin{tabular}{llcc}
\hline Correlación de variables & $\begin{array}{c}\text { rho } \\
\text { Spearman }\end{array}$ & de & P valor \\
\hline $\begin{array}{l}\text { Liderazgo transformacional } \\
\text { desarrollo sostenible verde }\end{array}$ & el & 0,788 & 0,000 \\
N & & 244 & 244 \\
\hline \multicolumn{2}{r}{} \\
\hline
\end{tabular}

Respecto a la prueba de hipótesis, como el diseño utilizado fue el correlacional, el estadígrafo utilizado fue el coeficiente de correlación rho de Spearman por tratarse de datos en escala ordinal, prueba bilateral a dos colas. Ahora bien, como la rho de Spearman $=0,788$ y el $p$-valor: 0,000 <0,005: entonces se rechaza la hipótesis nula

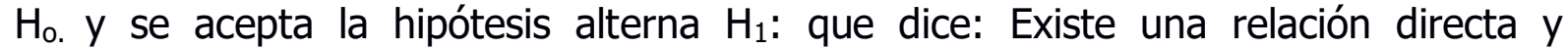
significativa entre el liderazgo transformacional y el desarrollo sostenible verde en la Universidad Nacional Hermilio Valdizán

En esta investigación se ha hallado en general que el tipo de relación entre el liderazgo transformacional y el desarrollo sostenible verde es directa y significativa en la Universidad Nacional Hermilio Valdizán. la corroborar estos resultados se encuentran algunos estudios relacionados como lo sostiene Cortés-Peña (2016) que sí existe la preocupación actitudinal de los jóvenes para fortalecer sus prácticas comportamiento proambiental e iniciar procesos de desarrollo económico sustentable.

Del mismo modo, los resultados obtenidos nos dicen que el nivel predominante en educación ambiental es medio o regular (Pérez-Franco, Pro-Bueno y Pérez-Manzano, 2018) citado en (Casa, Cusi y Vilca, 2019) afirman en su investigación que, la educación ambiental debe promover la conciencia ambiental, generando actitudes favorables a la protección del ambiente y al desarrollo sostenible, para contribuir estudiantes responsables con principios sólidos.

En verdad, el desarrollo sostenible verde, recae cuando las actitudes ambientales son predisposiciones del pensamiento del hombre para actuar de manera activa a favor o en contra del entorno, teniendo como base las vivencias, conocimientos y principalmente los valores que asume el ser humano sobre el entorno, resultado de procesos cognitivos, afectivos y conductuales. (Gonzáles, Machín y Galán, 2018).

Como mencionan (Piza-Flores, Aparicio, Rodríguez y Beltrán, 2018) la crisis ambiental planetaria demanda que las universidades cumplan su misión de formar 
Santacruz Espinoza, A., Montenegro Muguerza, H., Pizarro Alejandro, A. y Estacio Flores, H. Liderazgo transformacional y desarrollo sostenible ambiental verde en docentes de la Universidad Nacional Herminio Valdizán

egresados con las competencias necesarias para la atención de los temas emergentes sociales, dentro de ellos los referidos al medio ambiente, como una vía para llegar al desarrollo sustentable.

En el liderazgo transformacional, los líderes conmueven y cambian las acciones al comunicar e integrase en sus seguidores una visión y misión utilizando los ideales y motivos más convincentes para el cambio de actitud, así como para mitigar los problemas ambientales. Tal como lo sostiene (Fernando, 2015) el líder sostenible debe creer en su papel de transformador de la sociedad. La sustentabilidad y la responsabilidad social no pueden quedar solo en el ámbito empresarial. Se hace vital educar a todas las partes involucradas para generar una cultura relacionada con los temas de sustentabilidad. Por eso, para avanzar, la sustentabilidad requiere conciencia y conocimiento lo que transforma al líder sostenible en un agente protagonista de esta transformación. (...) este Este líder sostenible debe coordinar el proceso de cambio de mentalidad hacia la sustentabilidad, en la búsqueda de un desarrollo más equilibrado.

Mientras que, Gutiérrez (2017) da a conocer en su investigación sobre el concepto del ciclo del agua, el alumnado considera solamente aspectos evidentes y obvios en un proceso cerrado que no admite distintas perspectivas, no relacionan todos los posibles procesos físicos de cambio de estado ni conceptos como la escorrentía, aguas subterráneas, sublimación e infiltración debido a que son aspectos que no son tan evidentes en su cotidianidad. Además, la mayoría del alumnado cree que el ciclo natural del agua no está relacionado con el ciclo urbano del agua ni tienen en cuenta aspectos y/o efectos de los seres vivos.

En esta parte, Palavecinos, Amérigo, Ulloa y Muñoz (2016) describen la importancia de contar con instrumentos que permitan iniciar estudios en el contexto chileno en el área, considerando los factores contextuales, como la agudización de la crisis ambiental en el país, la preocupación ciudadana sobre la situación y la necesidad de realizar estudios transculturales para seguir profundizando en el conocimiento de la temática a nivel mundial.

Resulta evidente, que la comunidad universitaria-UNHEVAL participa de manera responsable, sostenible y solidaria con el desarrollo de las políticas públicas destinadas al logro del bien común y la justicia social para el fortalecimiento de la dignidad humana y respeto a la Naturaleza. Lo cual demanda a cada ser humano un compromiso ético, moral por parte de los estudiantes, docentes y administrativos de la Universidad Nacional Hermilio Valdizán.

Esta aseveración es corroborada por (Montenegro, 2019) cuando afirma que la naturaleza es la morada de todos, a ella debemos respetarla, cuidarla y protegerla para que nos siga ofreciendo sus bondades, para el buen vivir de todas y todos en el presente y las futuras generaciones. Practiquemos la reciprocidad, la solidaridad, la creatividad, el equilibrio, y el saber ser humano sensible.

En conclusión, docentes de la Universidad Nacional Hermilio Valdizán tuvieron un nivel medio o regular de en desarrollo sostenible verde, del mismo modo, se ha 
Santacruz Espinoza, A., Montenegro Muguerza, H., Pizarro Alejandro, A. y Estacio Flores, H. Liderazgo transformacional y desarrollo sostenible ambiental verde en docentes de la

Universidad Nacional Herminio Valdizán

percibido en ellos que dan orientación a la sociedad para el reciclado adecuado, propicia conversatorios para no pagar elevados precios por el consumo de electricidad o agua, comprar solo lo que se necesita, dejar de comprar alimentos o productos como una botella de agua de plástico contaminantes a la salud y al ambiente, utilizar el transporte de la universidad o público en vez de privado; ello, para la sostenibilidad generacional.

También, la comunidad universitaria manifestó que no están de acuerdo en pintar los troncos de los árboles, ya que estas terminan dañándolos con una capa tóxica al árbol. Lo cual contraviene a la economía ambiental sustentable. Por consiguiente, propusieron que en vez de pintarlos; abonarlos con fertilizantes orgánicos para que muestren su esplendor de su belleza natural, nos provean oxígeno para la sostenibilidad de la vida a través de la gestión de recursos para la economía circular, actividad económica e innovación ambiental verde. Pues, existe la necesidad de diseñar estrategias que incrementen la influencia desde los docentes para fortalecer la conducta ambiental entre sus estudiantes, y esta hacia la sociedad.

\section{REFERENCIAS}

Bartolotta, S. A. (2015). Daño en los ecosistemas y pérdida de biodiversidad, una delgada línea entre la inoperancia y la necesaria responsabilidad social. Argentina. Recuperado de https://www.oei.es/historico/divulgacioncientifica/?Dano-en-losecosistemas-y-perdida-de-biodiversidad-una-delgada-linea-entre-la

Bass, B. M. (1985). Leadership and performance beyond expectations. New York.

Bass, B., \& Avolio, B. (2006a). Manual for the multifactor leadership. California. Estados Unidos: Psychologist Press. Palo Alto

Becker, R. (2017). Indicadores de economía verde y de las ODS. Recuperado de https://tinyurl.com/yaqscbjm

Bernard, F. (1999). Ciencias ambientales, ecología y desarrollo sostenible. México: Interamericana.

Brack, A. (2012). Perú tiene mayor conciencia ambiental. Perú: Universia.

Brundtland, G. (1987). Nuestro futuro común. España: Naciones Unidas. Recuperado de https://tinyurl.com/ycathsxh; https://observatoriorsc.org/nuestro-futuro-comun/

Casa, M., Cusi, L. y Vilca, L. (2019). Percepciones sobre contaminación ambiental y actitudes en estudiantes universitarios. Innova Educación, 1(3), 390-399. doi: $\underline{10.35622 / j . r i e .2019 .03 .011}$

Chankrajang, T. y Muttarak, R. (2017). Green Returns to Education: Does Schooling Contribute to Pro-Environmental Behaviours? Evidence from Thailand, 131, 434448. doi: 10.1016/j.ecolecon.2016.09.015

Revista de Comunicación de la SEECI. 15 noviembre, 2020 / 15 marzo, 2021, nº 53, 135-151 
Santacruz Espinoza, A., Montenegro Muguerza, H., Pizarro Alejandro, A. y Estacio Flores, H. Liderazgo transformacional y desarrollo sostenible ambiental verde en docentes de la Universidad Nacional Herminio Valdizán

Drucker, P. (1993). Gerencia para el futuro. Colombia: Editorial Norma.

Escobedo, N. (2015). ISO 14001. Recuperado de https://www.youtube.com/watch?v=Tb29cZXwNvI

Fernando, M. (2015). La importancia del liderazgo sostenible como una estrategia de las organizaciones. Ciencias estratégicas, 23(34), 209-218. Recuperado de https://www.redalyc.org/articulo.oa?id=151350864004

Gardner, A. A. (2017). Sustainability Toolkit: An Education Tool for Behavioral Change Strategies. Recuperado de https://repository.arizona.edu/bitstream/handle/10150/625288/azu etd 15529 si p1 m.pdf?sequence $=1$ \&isAllowed $=y$

Goleman, D. (2013). Liderazgo. El poder de la inteligencia emocional. Recuperado de https://tinyurl.com/yayqwd78

Gonzáles, A., Machín, F. y Galán, V. (2018). Actitudes ambientales hacia la sostenibilidad agrícola desde la enseñanza de la física, 8(2), 17-31. doi: 10.35622/j.rie.2019.03.011

Gutiérrez, L. H. (2017). La educación ambiental: Una estrategia didáctica para favorecer e/o conocimiento escolar, Colombia. Recuperado de https://tinyurl.com/y8xjyvdp

Hwang, J., Park, Y. y Kim, Y. (2016). Why do consumers respond to eco-labels? The case of Korea. SpringerPlus, 5(1915). doi: 10.1186/s40064-016-3550-1

Jordán, R. y Garay, M. (2014). Liderazgo Real de los fundamentos a la práctica. Santiago: QuadGraphics.

Kerlinger, F. y Lee, H. (2002). Investigación del comportamiento. México: Interamericana S. A.

Lussier, R. y Achua, C. (2016). Liderazgo, teoría, aplicación en el desarrollo de habilidades. Recuperado de https://tinyurl.com/ycuzro2g

Lutz, W., Muttarak, R. y Striessnig, E. (2014). Universal education is key to enhanced climate adaptation. Science, 346 (6213), 1061-1062. doi: $\underline{10.1126 / \text { science. } 1257975}$

Meyer, A. (2015). Does education increase pro-environmental behavior? evidence. Ecological Economics, 108-121. doi: 10.1016/j.ecolecon.2015.04.018

Montenegro, H. (2019). Educación para el desarrollo sostenible del medio ambiente. Huánuco: Condorpasa. 
Santacruz Espinoza, A., Montenegro Muguerza, H., Pizarro Alejandro, A. y Estacio Flores, H. Liderazgo transformacional y desarrollo sostenible ambiental verde en docentes de la Universidad Nacional Herminio Valdizán

OCDE (2011). Hacia el crecimiento verde. Recuperado de https://www.oecd.org/greengrowth/49709364.pdf

Organización de las Naciones Unidas para la Alimentación y la Agricultura (2017). Objetivos del Desarrollo Sostenible. Recuperado de http://www.fao.org/sustainable-development-goals/mdg/goal-7/es/

Oseda, D., Santacruz, A., Zevallos, L. C., Sangama, J. L., Cosme, L. M. y Mendivel, K. (2018). Fundamentos de la investigación científica. Huancayo: Soluciones Gráficas.

Palavecinos, M., Amérigo, M., Ulloa, J. B. y Muñoz, J. (2016). Preocupación y conducta ecológica responsable en estudiantes universitarios: estudio comparativo entre estudiantes chilenos y españoles. Elservier, 143-148. doi: 10.1016/j.psi.2016.01.001

Pérez-Franco, D., Pro-Bueno, A. y Pérez-Manzano, A. (2018). Actitudes ambientales al final de la ESO. Un estudio diagnóstico con alumnos de secundaria de la Región de Murcia. Eureka sobre Enseñanza y Divulgación de las Ciencias, 15(3), 350117. Recuperado de https://www.redalyc.org/articulo.oa?id=920/92054992014

Piza-Flores, V., Aparicio, J. L., Rodríguez, C. y Beltrán, J. (2018). Transversalidad del eje "Medio ambiente" en educación superior: un diagnóstico de la Licenciatura en Contaduría de la Universidad Autónoma de Guerreroo. Iberoaméricana para la Investigación y el Desarrollo Educativo, 8(16), 7-24. doi: 10.23913/ride.v8i16.360

Pozueta, F. (2015). Liderazgo emocional. Obtenido de: https://tinyurl.com/y8x9lfpj

Real Academia de la Lengua Española (2018). Liderazgo. Obtenido de: https://www.rae.es/

\section{AUTORES:}

\section{Atanacia Santacruz Espinoza}

Es docente a tiempo completo de la Universidad nacional Intercultural de la Amazonia. Licenciada en Educación, Maestría en Investigación y Docencia Superior (universidad nacional Hermilio Valdizán de Huánuco, Perú), Doctorado en Ciencias de la Educación. Ha realizado estudios de Segunda Especialización en Educación Inicial. Ha sido Jefe de Departamento de Humanidades de 2010-2012. Ha sido asesora de Pregrado de la UNIA, y Doctorado de la Universidad Nacional Hermilio Valdizán. publicaciones arbitradas a nivel nacional e internacional.

atanacia.santacruz@gmail.com

Orcid ID: https://orcid.org/0000-0002-3103-8947

\section{Humberto Montenegro Muguerza}

Posdoctorado en Ciencias. Doctor en Educación y Doctor en Derecho en la Universidad Nacional Hermilio Valdizán. Licenciado en Educación, Especialidad Leguaje y Filosofía. También obtuvo el Título Profesional en Derecho y Ciencias 
Santacruz Espinoza, A., Montenegro Muguerza, H., Pizarro Alejandro, A. y Estacio Flores, H. Liderazgo transformacional y desarrollo sostenible ambiental verde en docentes de la Universidad Nacional Herminio Valdizán

Políticas, ambas licenciaturas se obtuvieron en la Universidad Nacional Hermilio Valdizán. Docente ordinario en la UNHEVAl. Docente invitado a nivel de Posgrado en la UNHEVAL, César Vallejo, Universidad Nacional Agraria de la Selva, impartió las disciplinas científicas de Investigación Científica, Lingüística, Filosofía del Derecho, Derecho Constitucional... Conferencista en las Universidades de: Huánuco, Tingo María, Lima, Chiclayo y Cajamarca; Internacional: Universidad Autónoma de Madrid, España, en la Universidad del Valle, Colombia. Además, es poeta, músico y declamador. Pertenece a la Asociación Peruana de Literatura Infantil y Juvenil (APLIJ).

catedraHMM 1@hotmail.com

Orcid ID: https://orcid.org/0000-0002-2479-5587

\section{Armando Pizarro Alejandro}

Es docente de la Universidad Nacional Hermilio Valdizán de Huánuco, en la Facultad de Derecho y Ciencias Políticas, Maestría en Derecho, con Mención en Ciencias Penales (Universidad Nacional Hermilio Valdizán de Huánuco, Perú), Doctorado en Derecho. Ha realizado estudios Filosofía y Ciencias Sociales. Actualmente Decano de la Carrera Profesional de Derecho y Ciencias Políticas de la Universidad Nacional Hermilio Valdizán. Ha sido asesor de Pregrado, Maestría y Doctorado de la Universidad Nacional Hermilio Valdizán.

pizarro1905@hotmail.com

Orcid ID: https://orcid.org/0000-0003-2988-8085

\section{Hamilton Estacio Flores}

Abogado egresado de la Universidad Nacional Mayor de San Marcos; Magister en Derecho, con mención en Derecho Civil y Comercial, por la Universidad Nacional Hermilio Valdizán; Bachiller en Derecho y Ciencias Políticas, por la Universidad Nacional Mayor de San Marcos; Doctor en Derecho, por la Universidad Nacional Hermilio Valdizán; conferencista a nivel nacional e internacional. Actualmente es Director de la Escuela de Posgrado de la Facultad de Derecho y Ciencias Políticas. hamilton.e.s@hotmail.com

Orcid ID: https://orcid.org/0000-0002-8735-3591 